Cite this: Phys. Chem. Chem. Phys. 2014, 16, 17253

Received 27th May 2014, Accepted 5th June 2014

DOI: $10.1039 / \mathrm{c} 4 \mathrm{cp} 02322 \mathrm{f}$

www.rsc.org/pccp

\section{Use of side-chain for rational design of n-type diketopyrrolopyrrole-based conjugated polymers: what did we find out? $\dagger$}

\author{
Catherine Kanimozhi, ${ }^{a}$ Nir Yaacobi-Gross, ${ }^{b}$ Edmund K. Burnett, ${ }^{c}$ \\ Alejandro L. Briseno, ${ }^{c}$ Thomas D. Anthopoulos, ${ }^{b}$ Ulrike Salzner ${ }^{d}$ and Satish Patil ${ }^{\star a}$
}

\begin{abstract}
The primary role of substituted side chains in organic semiconductors is to increase their solubility in common organic solvents. In the recent past, many literature reports have suggested that the side chains play a critical role in molecular packing and strongly impact the charge transport properties of conjugated polymers. In this work, we have investigated the influence of side-chains on the charge transport behavior of a novel class of diketopyrrolopyrrole (DPP) based alternating copolymers. To investigate the role of sidechains, we prepared four diketopyrrolopyrrole-diketopyrrolopyrrole (DPP-DPP) conjugated polymers with varied side-chains and carried out a systematic study of thin film microstructure and charge transport properties in polymer thin-film transistors (PTFTs). Combining results obtained from grazing incidence $X$-ray diffraction (GIXD) and charge transport properties in PTFTs, we conclude side-chains have a strong influence on molecular packing, thin film microstructure, and the charge carrier mobility of DPP-DPP copolymers. However, the influence of side-chains on optical properties was moderate. The preferential "edge-on" packing and dominant $\mathrm{n}$-channel behavior with exceptionally high field-effect electron mobility values of $>1 \mathrm{~cm}^{2} \mathrm{~V}^{-1} \mathrm{~s}^{-1}$ were observed by incorporating hydrophilic (triethylene glycol) and hydrophobic side-chains of alternate DPP units. In contrast, moderate electron and hole mobilities were observed by incorporation of branched hydrophobic side-chains. This work clearly demonstrates that the subtle balance between hydrophobicity and hydrophilicity induced by side-chains is a powerful strategy to alter the molecular packing and improve the ambipolar charge transport properties in DPP-DPP based conjugated polymers. Theoretical analysis supports the conclusion that the side-chains influence polymer properties through morphology changes, as there is no effect on the electronic properties in the gas phase. The exceptional electron mobility is at least partially a result of the strong intramolecular conjugation of the donor and acceptor as evidenced by the unusually wide conduction band of the polymer.
\end{abstract}

\section{Introduction}

Charge transport properties of $\pi$-conjugated polymers are affected by many factors, such as regioregularity, molecular weight, crystallinity, nature of the alkyl chains, and their fabrication into thin films for use in electronic devices. The length and branching of side-chains has been found to have a profound impact on

\footnotetext{
${ }^{a}$ Solid State and Structural Chemistry Unit, Indian Institute of Science, Bangalore 560012, India. E-mail: satish@sscu.iisc.ernet.in; Fax: +91-80-23601310; Tel: $+91-80-22932651$

${ }^{b}$ Department of Physics and Centre for Plastic Electronics, Blackett Laboratory, Imperial College London, London SW7 2BW, UK

${ }^{c}$ Department of Polymer Science and Engineering, University of Massachusetts, Amherst, Massachusetts 01003, USA

${ }^{d}$ Department of Chemistry, Bilkent University, 06800 Bilkent, Ankara, Turkey $\dagger$ Electronic supplementary information (ESI) available: Materials, experimental details, additional spectra and device fabrication. See DOI: $10.1039 / \mathrm{c} 4 \mathrm{cp} 02322 \mathrm{f}$
}

charge transport behaviour. ${ }^{1}$ Traditionally, side-chains were introduced to impart solubility in $\pi$-conjugated polymers. ${ }^{2-5}$ The increased solubility of polymers improves the processability of thin films in common organic solvents and leads to high degree of polymerization during the synthesis of polymers. ${ }^{6,7}$ Recently, tremendous efforts have been made to understand the role of side-chains on the molecular packing and charge-carrier transport properties of $\pi$-conjugated polymers in thin-film transistors. ${ }^{8,9}$ The engineering of side-chains has guided synthetic chemists to design new materials with exceptionally high charge-carrier mobilities. Carrier mobilities of $\sim 10 \mathrm{~cm}^{2} \mathrm{~V}^{-1} \mathrm{~s}^{-1}$ have been achieved in p-type polymer thin-film transistors (PTFTs) for solution processable conjugated polymers. ${ }^{10}$ However, airstable high mobility n-type organic semiconductors are rare, preventing the practical application of flexible electronics to ambipolar transistors, complementary circuits, and organic photovoltaic devices. ${ }^{11}$ 
Such uneven development of p-type semiconductors compared to n-type semiconductors shows the high sensitivity and low stability of these materials. This is due to the serious drawbacks, including the stability of the anions with respect to atmospheric oxygen and water. ${ }^{12,13}$ Even though the factors governing the stability of n-type materials are not yet well understood, the first redox potential $\left(E_{\text {red1 }}\right)$ of the n-type semiconductor can be related to its capability to operate in ambient conditions. ${ }^{14}$

According to the de Leeuw's hypothesis, ${ }^{15}$ the first reduction potential of n-type semiconductor should be greater than $-0.66 \mathrm{~V}$ vs. SCE (which is the reduction potential of $\mathrm{H}_{2} \mathrm{O}$ ) in order to avoid the oxidation of reduced anions by $\mathrm{H}_{2} \mathrm{O}$ as shown in Fig. 1. Meanwhile a reduction potential greater than $+0.57 \mathrm{~V} v s$. SCE would be required to prevent the oxidation of polymeric anions by oxygen. Under experimental conditions, however, significant over potentials $(0.5 \mathrm{~V})$ are expected; therefore positive

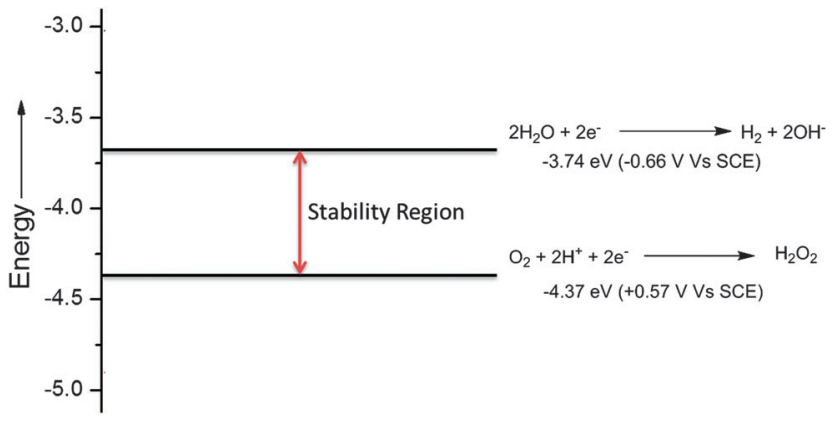

Fig. 1 Electrochemical stability window of $n$-type semiconductors. electrode potential is required for the electrochemical stability of n-type materials under ambient conditions.

This relationship has been further supported by the observation that the $n$-channel organic thin-film transistor (OTFT) devices recover their activity when re-measured under vacuum, after ambient exposure. ${ }^{16}$ In view of these facts, it is surprising to observe n-channel OTFTs at ambient conditions. However, through appropriate synthetic routes and rational molecular design, several n-type molecular and polymeric semiconductors have been synthesized exhibiting OTFT electron mobilities of $0.1-2.0 \mathrm{~cm}^{2} \mathrm{~V}^{-1} \mathrm{~s}^{-1}$ under inert/nitrogen atmosphere as well as under ambient atmosphere. ${ }^{16-20}$

By satisfying de Leeuw's hypothesis, our group has reported a high electron mobility of $3 \mathrm{~cm}^{2} \mathrm{~V}^{-1} \mathrm{~s}^{-1}$ for a diketopyrrolopyrrolediketopyrrolopyrrole based conjugated polymer. ${ }^{21}$ The polymer was substituted with hydrophobic and hydrophilic side-chains on alternate units of DPP. The observation of such high electron mobility is striking, although we rationally coupled DPP to DPP to reduce the LUMO energy to enhance the stability of the anion towards water and oxygen. To understand the origin of the high electron mobility and molecular packing, and the role of side-chains in the intermolecular interactions and polymer electrical properties in OTFTs, in the present work, we have designed and synthesized three new DPP-DPP copolymers bearing side chains with varying chain lengths and hydrophobic/hydrophilic groups. Significant efforts were also devoted to understand the role of side-chains in the solution processability, molecular packing and thin film microstructure. Chemical structures of copolymers 2DPP-OD-TEG, 2DPP-OD-HE, 2DPP-OD-EH and 2DPP-OD-OD are shown in Fig. 2.
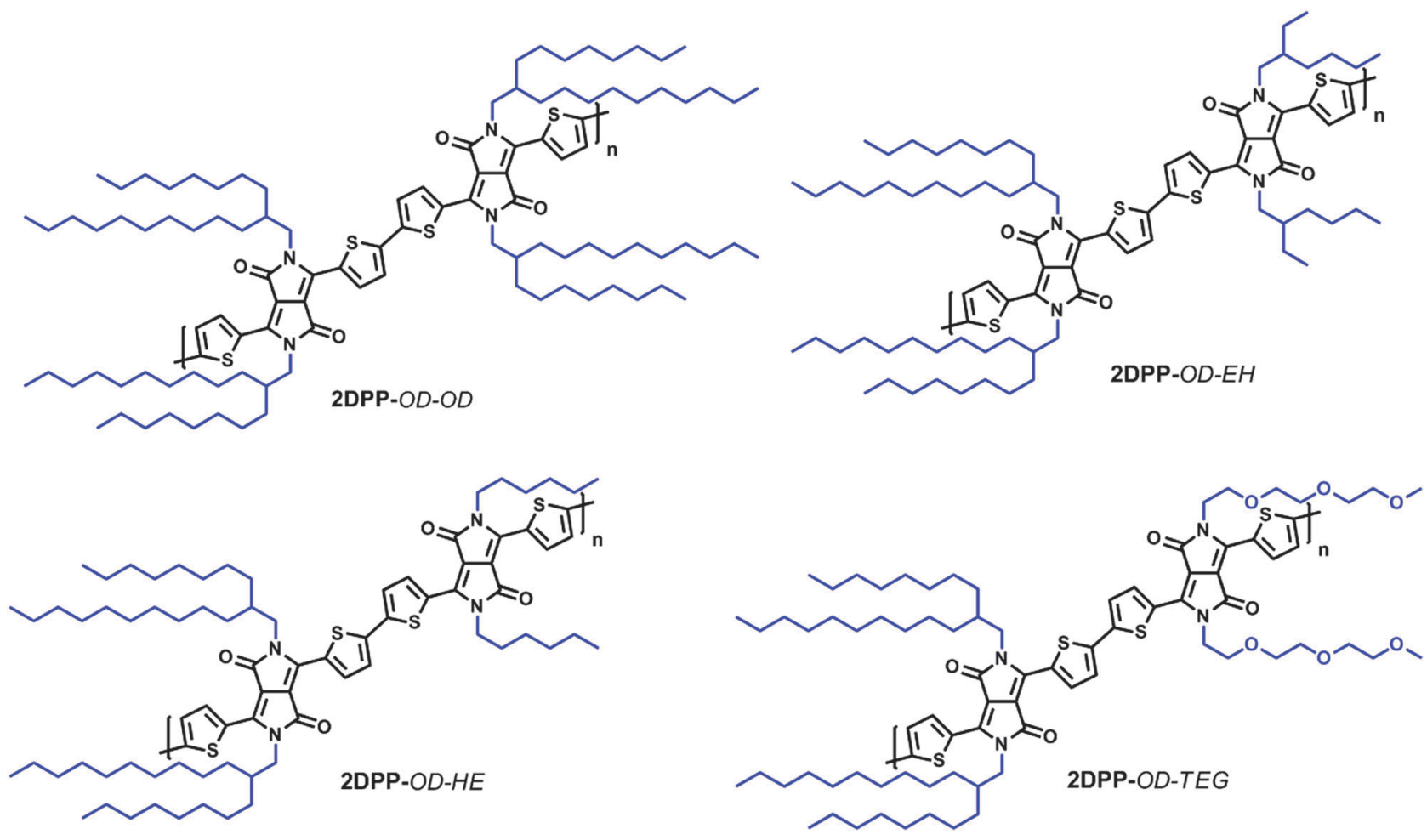

Fig. 2 Chemical structure of DPP-DPP copolymers. 
Our design principle for the synthesis of DPP-DPP copolymers with different alkyl/ethylene glycol side-chains serves to gain deeper insight into the role of alkyl chains on the lactam unit of DPP, with a broader objective of understanding the basic relationship between structural features and performance characteristics of the newly synthesized polymers. The bulkier alkyl chain could result in a larger twist angle and a less planar geometry, resulting in unfavourable steric interactions with adjacent conjugated subunits and leading to poor electronic communication between the donor and acceptor units, ${ }^{22}$ which in turn can affect the intramolecular ${ }^{23}$ charge transfer along the conjugated backbone. ${ }^{24}$ This understanding can be effectively utilized to identify and modify the inherent properties of the molecular semiconductors in terms of their spectral width, band gap, thin film morphology and processing conditions. The important outcome from this study is the ambipolar charge transport and influence of hydrophilic chains on the charge transport properties of DPP-DPP based copolymers.

\section{Results and discussions}

\section{Synthesis and characterization}

The synthetic route for the monomers and copolymers is outlined in Schemes 1 and 2. Compounds 1a-d and monomers M1-M5 were synthesized by analogous procedures to those previously reported in the literature. ${ }^{25,26}$ A detailed description of the synthesis of monomers is given in the ESI. $\dagger$ The key monomer M1 was prepared from lithium diisopropylamide (LDA) and 2-isopropoxy-4,4,5,5-tetramethyl-1,3,2-dioxaboralane with small modifications to the literature procedure. All the monomers were obtained in good yield, purified by column

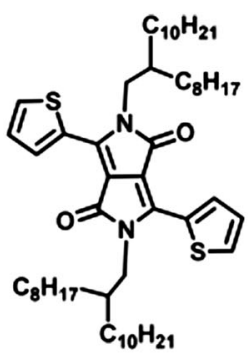<smiles>CC(C)OB1OC(C)(C)C(C)(C)O1</smiles><smiles>[R]N1C(=O)C2=C(c3cccs3)N([R])C(=O)C2=C1c1cccs1</smiles>

N- bromosuccinimide

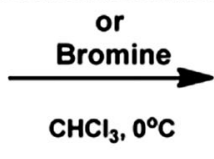

M1
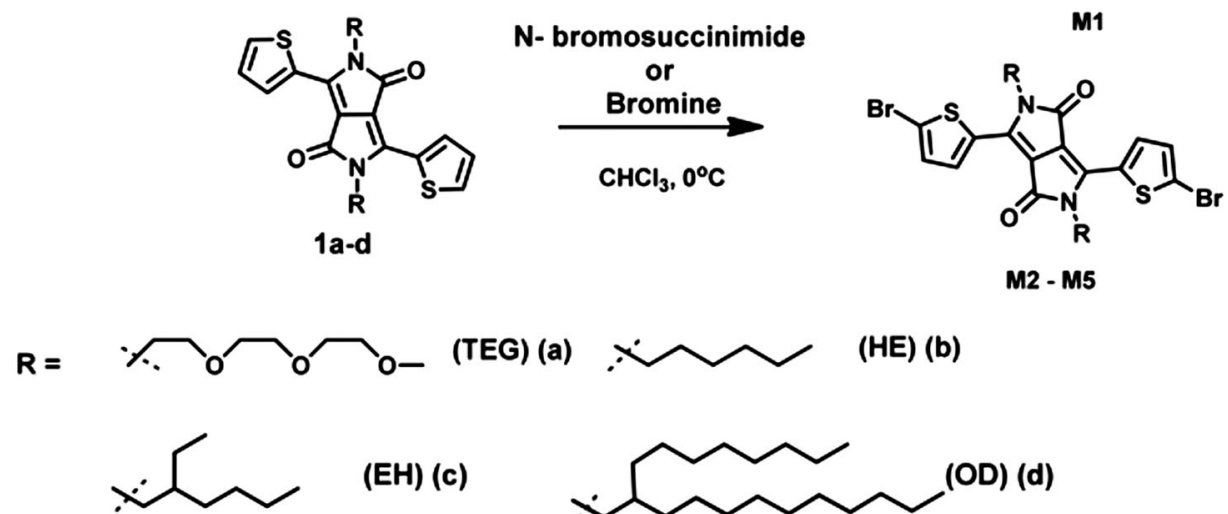

(TEG) (a)

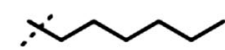

(HE) (b)

(EH) (c)

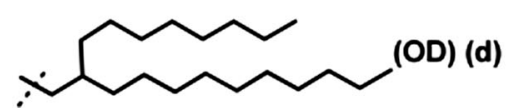

Scheme 1 Synthetic pathway for the monomers M1-M5.

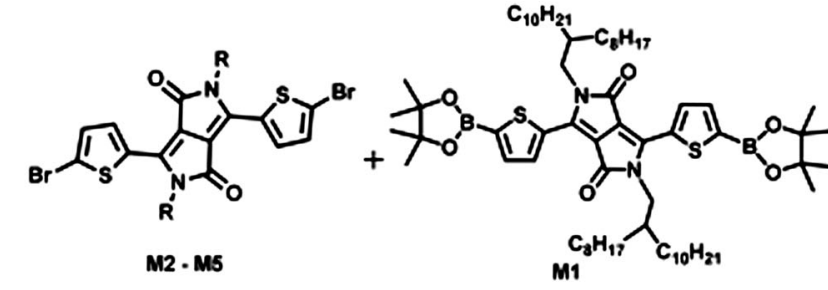

$\mathbf{R}=$

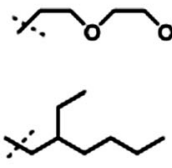

(EH)
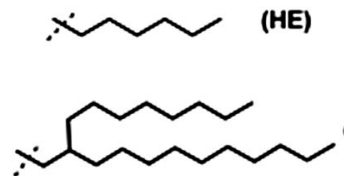

(OD)

Scheme 2 Synthesis of DPP-DPP copolymers by Suzuki coupling reaction. 
chromatography and characterized by ${ }^{1} \mathrm{H},{ }^{13} \mathrm{C}$ NMR and elemental analysis. The DPP-DPP copolymers were synthesized via palladium-catalyzed Suzuki cross-coupling reactions between 3,6-bis(5-(4,4,5,5-tetramethyl-1,3,2-dioxaborolan-2-yl)thiophen-2yl)- $N, N$-bis(2-octyldodecyl)-1,4-dioxopyrrolo[3,4-c]pyrrole (M1) and 3,6-bis(5-bromothiophen-2-yl)- $N, N$-dialkyl-1,4-dioxopyrrolo[3,4-c]pyrrole (M2-M5). The polymerization reaction was carried out in the presence of a metal precursor catalyst $\operatorname{Pd}_{2}(\mathrm{dba})_{3}$ and an active ligand tri $(o$-tolyl $)$ phosphine $\left(\mathrm{P}(o \text {-tol })_{3}\right)$. After completion of the polymerization, solvent was removed and the polymers were purified by precipitation in acetone and followed by Soxhlet extraction with methanol, acetone and hexane.

Table 1 Optical properties and molecular weights of DPP-DPP polymers

\begin{tabular}{|c|c|c|c|c|c|c|c|}
\hline \multirow[b]{3}{*}{ Polymer } & \multicolumn{4}{|c|}{ UV-Vis absorption } & \multicolumn{3}{|c|}{ Molecular weight data } \\
\hline & \multirow{2}{*}{$\begin{array}{l}\text { Solution } \\
\lambda_{\max } \\
(\mathrm{nm})\end{array}$} & \multicolumn{2}{|c|}{ Thin film } & \multirow[b]{2}{*}{$\begin{array}{l}E_{\mathrm{g}}^{\mathrm{opt}} \\
(\mathrm{eV})\end{array}$} & \multirow[b]{2}{*}{$\begin{array}{l}M_{\mathrm{n}}(\mathrm{kg} \\
\left.\mathrm{mol}^{-1}\right)\end{array}$} & \multirow[b]{2}{*}{$\begin{array}{l}M_{\mathrm{w}}(\mathrm{kg} \\
\left.\mathrm{mol}^{-1}\right)\end{array}$} & \multirow{2}{*}{$\begin{array}{l}\text { PDI } \\
M_{\mathrm{w}} / \\
M_{\mathrm{n}}\end{array}$} \\
\hline & & $\begin{array}{l}\lambda_{\max } \\
(\mathrm{nm})\end{array}$ & $\begin{array}{l}\lambda_{\text {onset }} \\
(\mathrm{nm})\end{array}$ & & & & \\
\hline 2DPP-OD-HE & 924 & 939 & 1020 & 1.21 & 13.2 & 93.2 & \\
\hline 2DPP-OD-E & 924 & 948 & 1025 & 1.20 & 13.3 & 100.2 & \\
\hline 2DPP-OD-OD & 898 & 920 & 1010 & 1.22 & 20.8 & 25.5 & \\
\hline 2DPP-OD-TEG & 905 & 951 & 1023 & 1.21 & 67.3 & 314.0 & \\
\hline
\end{tabular}

${ }^{a}$ Determined by SEC in trichlorobenzene and in chloroform based on polystyrene standards. The molecular weight for 2DPP-OD-TEG is also reported in ref. 21.
GPC analysis of the copolymers was carried out in different solvents at elevated temperature. The average molecular weights $\left(M_{\mathrm{w}}\right.$ and $\left.M_{\mathrm{n}}\right)$ and polydispersity indices (PDI) were obtained against a polystyrene standard and the results are summarized in Table 1. The results obtained from GPC analysis showed unusually high polydispersity and a bimodal distribution. The low solubility of the polymers in common organic solvents leads to aggregation at room temperature, which severely affects the observed polydispersity of such conjugated polymers. Similar aggregation behavior has been observed in many DPP based conjugated polymers even at high temperatures. ${ }^{27-29}$

\section{Electronic structure and optical properties}

The copolymers designed in this work, with the DPP-DPP as a strongly electron-deficient unit along the polymer chain, exhibit unique optical and electronic properties. The optical absorption spectra of 2DPP-OD-TEG, 2DPP-OD-HE, 2DPP-OD-EH and 2DPPOD-OD in chloroform and in thin film are shown in Fig. 3 and significant optical properties are summarized in Table 1 . The absorption spectra of these copolymers in solution and in thin film exhibit a vibronically structured band with an onset at $1000 \mathrm{~nm}$. The spectrum shows typical characteristics of homopolymers rather than alternating donor-acceptor copolymers. The striking difference noted in this family of DPP-DPP polymers is the huge red shift $>300 \mathrm{~nm}$ and high oscillator strength of the low energy band with an exceptionally high
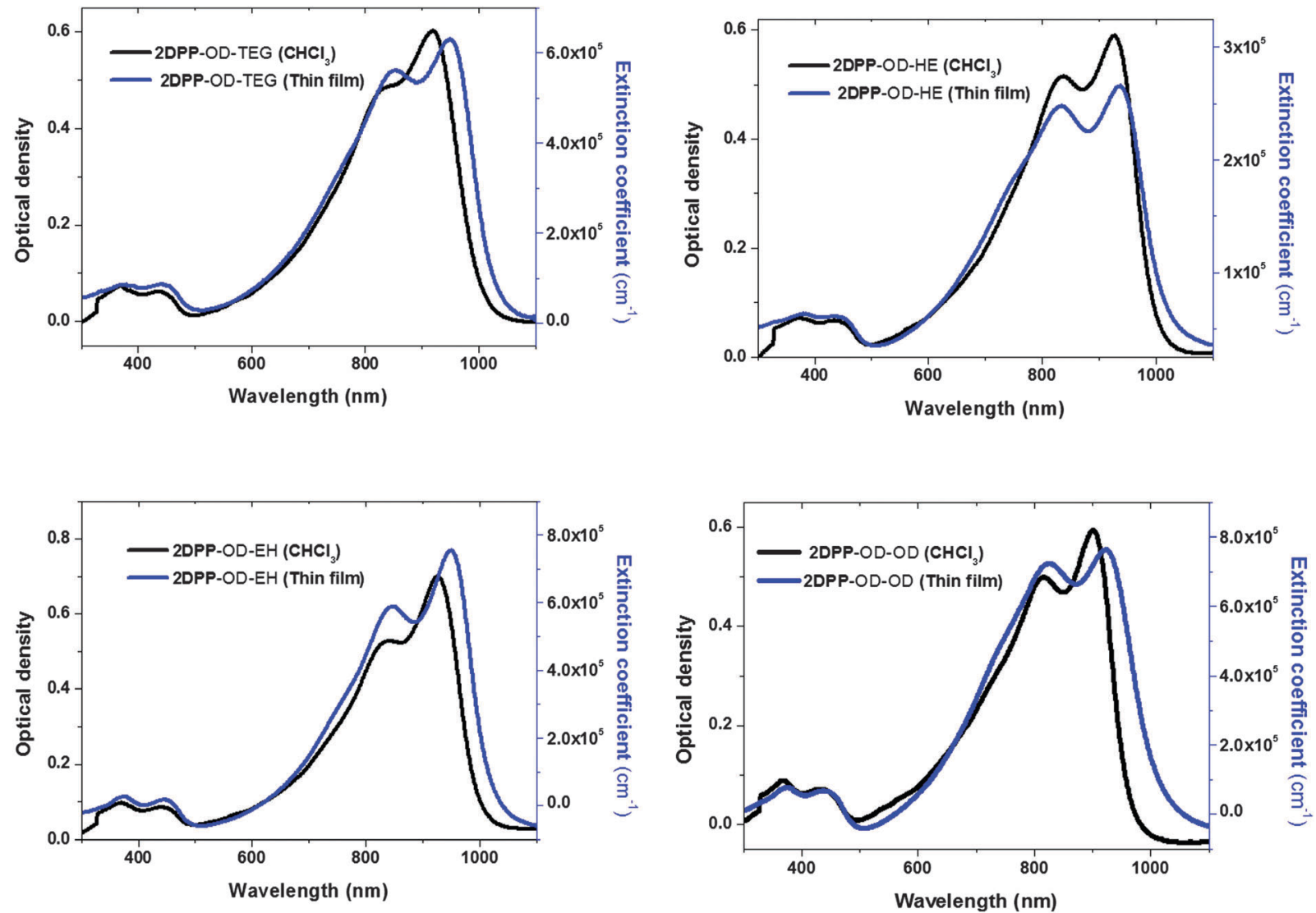

Fig. 3 UV-Visible absorption spectra of DPP-DPP copolymers in chloroform and in thin film. 
molar extinction coefficient, while the intensity of the band observed at $400 \mathrm{~nm}$ is very low. The high oscillator strength and red shift of the low energy band suggests a longer conjugation length caused by the strong intramolecular interactions of the donor-acceptor repeating unit of polymer backbone, that was enhanced by an increased degree of coplanarity. Such enhanced intramolecular charge transfer in low band-gap polymers and oligomers has been found to improve the charge carrier mobility in OTFT. ${ }^{30,31}$ The optical band-gaps ( $E_{\mathrm{g}}^{\text {opt }}$ ) of these copolymers were estimated by extrapolating the onset of absorption edge and were found to be $\sim 1.2 \mathrm{eV}$. The thin film absorption spectra of 2DPPOD-TEG, 2DPP-OD-HE, 2DPP-OD-EH and 2DPP-OD-OD exhibit dramatic red shifts of $46 \mathrm{~nm}, 15 \mathrm{~nm}, 24 \mathrm{~nm}$ and $22 \mathrm{~nm}$ respectively, suggesting the type of alkyl chain has a strong influence on conformational changes of the polymer backbone, which leads to strong intermolecular interactions in the solid state.

The redox potentials and the HOMO-LUMO energy levels of the polymers have been determined electrochemically by cyclic voltammetry (CV) and the data is given in the ESI $\dagger$ (Fig. S2 and Table S1). All of the four polymers show remarkable stability during the reduction cycle of $\mathrm{CV}$. The representative $\mathrm{CV}$ of multiple cycles for 2DPP-OD-TEG is given in ESI $\dagger$ (Fig. S3). The introduction of the electron deficient DPP-DPP unit in the polymer backbone stabilizes the anion introduced during electrochemical reaction. Additionally, the LUMO levels of conjugated polymers drastically shift to deeper values as compared to conventional DPP-based copolymers such as DPP-benzothiadiazole and DPP-thienothiophene. This suggests that the strong overlap of donor-acceptor unit resulting from the coplanarity can result in substantial changes in energy levels, electrochemical and optical properties.

\section{Thermal properties}

The thermal stability of these copolymers was investigated by thermal gravimetric analysis (TGA) and the data is given in the ESI. $\dagger$ The thermal stability of molecular semiconductors is an important parameter. Thin films are often exposed to harsh environments during device fabrication, such as thermal annealing during the evaporation of the top electrode. TGA analysis of the copolymers indicates stability above $350{ }^{\circ} \mathrm{C}$ with an observed decomposition temperature in the range of $360-380{ }^{\circ} \mathrm{C}$ (Fig. S1, ESI $\dagger$ ).

\section{Thin film morphology}

The thin film morphology and roughness of the semiconductor layer is critical in improving the performance of OTFTs and OPVs. A large donor-acceptor interface is required to ensure efficient charge dissociation, while trap free percolating pathways are essential to allow efficient charge transport. It is necessary to control the morphology of the active layer by various processing methods such as thermal and solvent annealing, or using mixed solvent systems. The nature of the polymer backbone and alkyl chains also have great influence on the morphology of the polymer thin film. Atomic force microscopy (AFM) and field emission scanning electron microscopy (FESEM) were employed to evaluate the thin film surface morphology of the copolymers.

AFM images of the copolymers spin coated or drop-casted from $o$-dichlorobenzene are shown in Fig. 4. Prior to a deposition, the polymer solutions were sonicated and then passed through a membrane filter (pore diameter of $0.2 \mu \mathrm{m}$ ). Copolymers with linear, branched and hydrophobic/hydrophilic side-chains (2DPP-OD-HE, 2DPP-OD-EH and 2DPP-OD-TEG) resulted in a
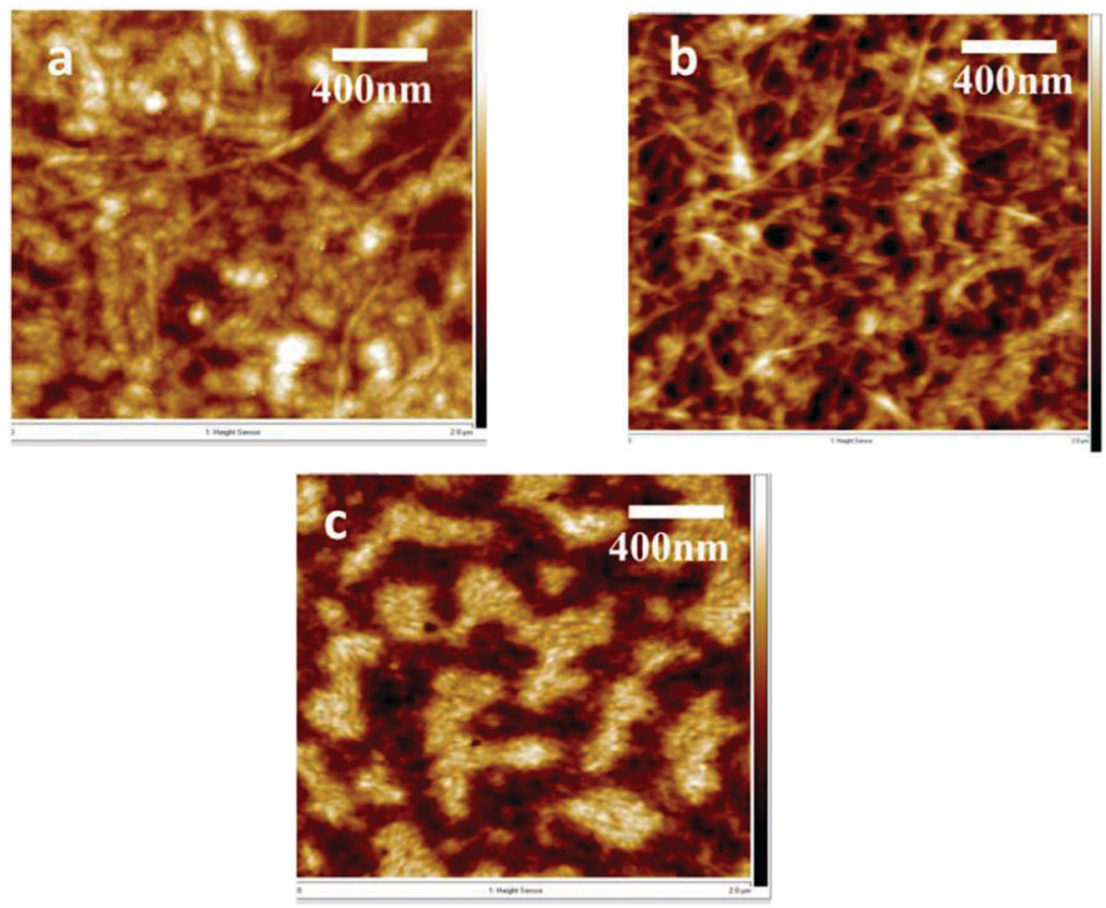

Fig. 4 AFM height images of (a) 2DPP-OD-HE, (b) 2DPP-OD-EH and (c) 2DPP-OD-TEG spin coated from o-dichlorobenzene. 
fibrillar morphology composed of nanofibers. In contrast, the copolymer with branched 2-octyldodecyl chains (2DPP-OD-OD) lacked fiber-like features. The variation of the length and nature of side-chains results in a clear transition of well-defined fiberlike structures to a featureless morphology. This clearly indicates the strong influence of side chains on polymer conformations. The presence of strong intermolecular interactions such as van der Waals, $\pi-\pi$ stacking and difference in solubility is evident from the formation of structural morphology.

FESEM images of the copolymers were obtained from o-dichlorobenzene (ODCB) as shown in Fig. 5. As observed in the AFM images of the polymers, a similar nanofibrillar morphology was observed for copolymers 2DPP-OD-TEG, 2DPP-OD-HE, and 2DPP-OD-EH when cast from ODCB. Dispersed and entangled features of nanofibers were observed for 2DPP-OD-HE and 2DPP-OD-EH. In contrast, 2DPP-OD-TEG exhibited long range nanofibrils structures with improved alignment. The width of nanofibers varies with diameters of $40-100 \mathrm{~nm}$ as we change the nature and length of side-chains. 2DPP-OD-TEG exhibited nanofibers with a diameter of $40 \mathrm{~nm}$, whereas 2DPP-OD-HE and 2DPP-OD-EH showed nanofibers with a diameter of 100 and $\sim 130 \mathrm{~nm}$ respectively. We have not observed fibrillar-like morphology for 2DPP-OD-OD. These results clearly suggest that the hydrophilic triethylene glycol side chains promote the reorganization of polymer chains into more ordered aggregates, and such structures have been proven beneficial for charge carrier transport and improved device performance. Unlike the polymers with linear hydrophobic/hydrophilic chains or smaller branched chains, the polymer with longer branched alkyl chains (2DPP-OD-OD) showed no structures, instead resulting in a thin film as shown in Fig. 5(d). The fibrillar morphology of the polymers with short and linear alkyl chains clearly indicates the presence of favorable intermolecular forces present in the bulk polymer film, and indeed the insulating alkyl chains affect the solid state packing of DPP-based conjugated polymers.

\section{Thin film X-ray diffraction studies}

The effect of side chains on the thin film microstructures and the orientation of polymer crystallites on substrates was investigated by wide angle X-ray diffraction (XRD). The thin films of copolymers were cast from chlorobenzene onto silicon substrates. The copolymers 2DPP-OD-HE and 2DPP-OD-TEG (Fig. 6) with linear alkyl chains exhibit intense low angle Bragg peaks at $2 \theta=4.35^{\circ}$, attributed to the lamellar packing with an intermolecular distance of $20.2 \AA$, and a peak at $2 \theta=22^{\circ}$ corresponding to the $\pi-\pi$ stacking interactions between the polymer conjugated planar backbones with a $d$-spacing in the order of $4.0 \AA$.

Copolymers with branched alkyl chains such as 2DPP-OD-EH and 2DPP-OD-OD exhibit reduced crystallinity or amorphous nature. Copolymer 2DPP-OD-OD with repetitive dodecyl alkyl chains did not show small angle peaks, implying that the longer branched dodecyl alkyl chains prevent strong solid state packing.

Although wide angle XRD is a powerful tool to probe the overall crystallinity of macromolecules, it is difficult to identify
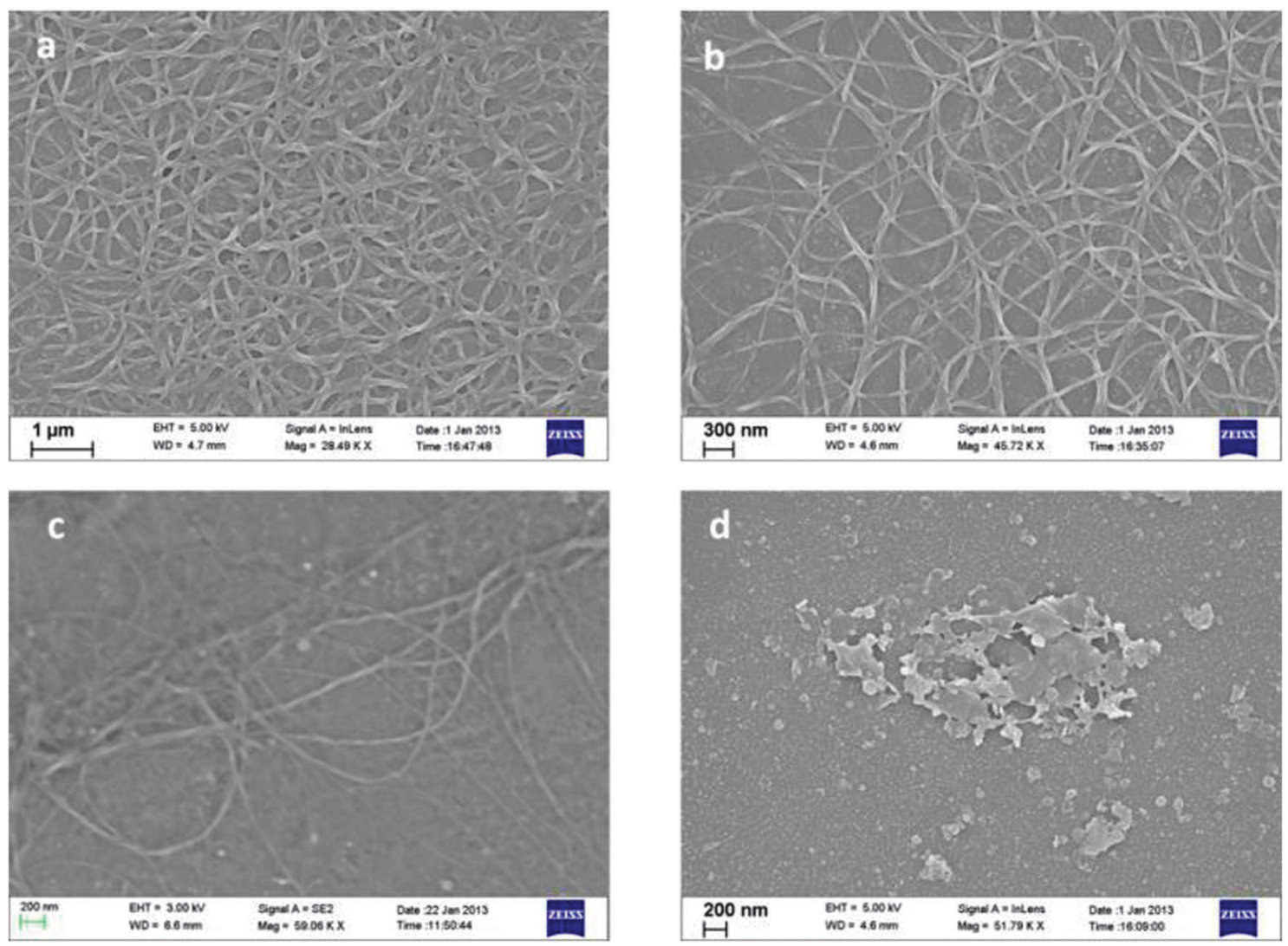

Fig. 5 FESEM images of (a) 2DPP-OD-HE, (b) 2DPP-OD-EH, (c) 2DPP-OD-TEG and (d) 2DPP-OD-OD drop cast from ODCB. 

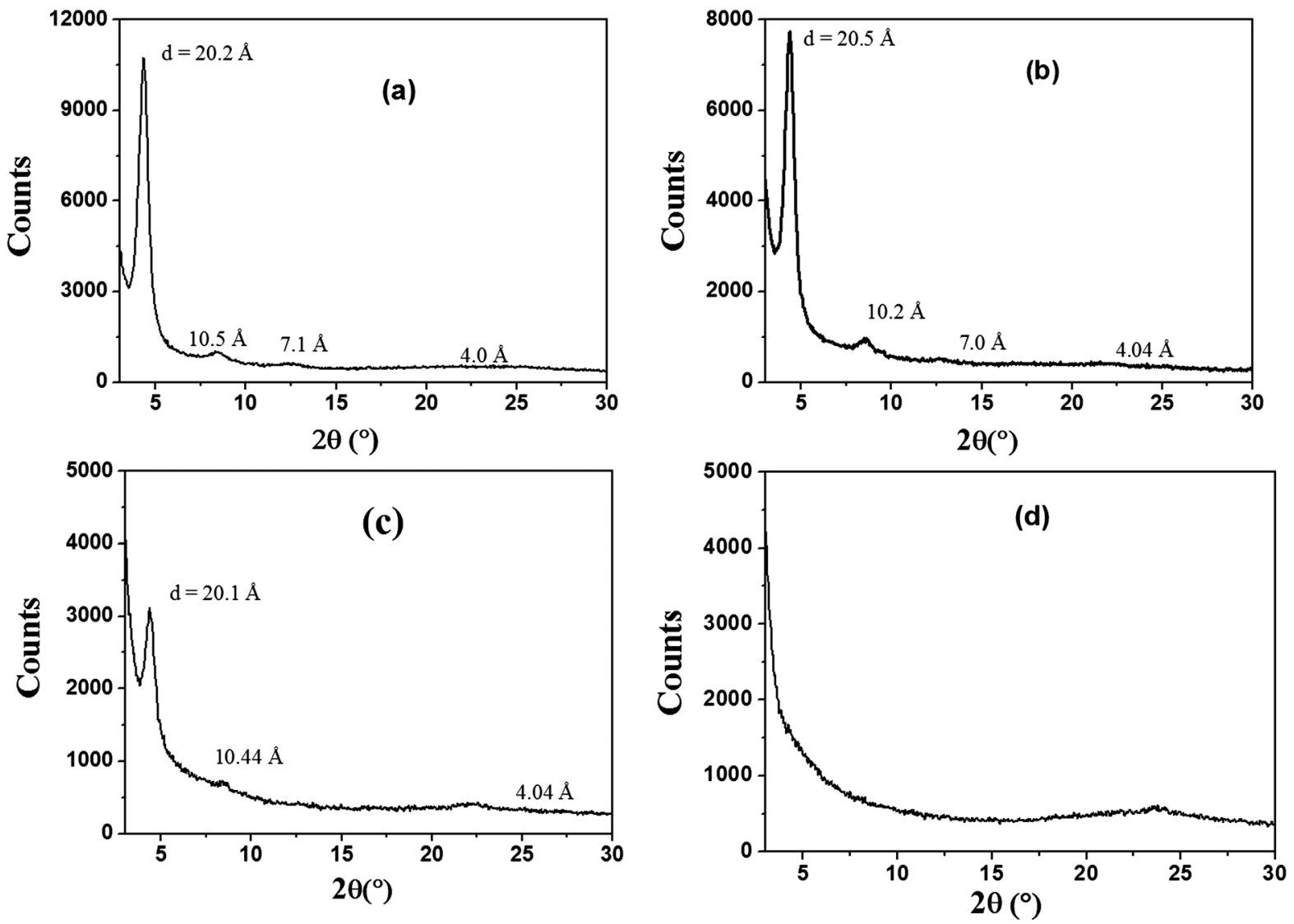

Fig. 6 Thin film X-ray diffraction of DPP-DPP copolymers (a) 2DPP-OD-TEG, (b) 2DPP-OD-HE, (c) 2DPP-OD-EH and (d) 2DPP-OD-OD on a Si substrate.

the mixed phases present in the thin film and the molecular packing of conjugated polymers, especially in the out-of-plane $\left(q_{z}\right)$ and in-plane $\left(q_{x y}\right)$ directions of the polymer backbone. These are crucial with respect to the charge transport properties of PTFT. We carried out grazing-incidence X-ray diffraction (GIXD) experiments to extract the orientation of the polymer crystallites by casting polymer thin films on Si substrates.

Fig. 7 shows the GIXD data and the intensity profile for as spun 2DPP-OD-TEG. The high order lamellar structures $(h 00)$ parallel to the normal of the surface are clearly evident. A lamellar $d$-spacing of $20 \AA$ was determined from the first-order (100) peak, which has a coherence length of $91 \AA$ A Similarly an in-plane $\pi-\pi$ stacking (010) peak associated with a $d$-spacing of $3.6 \AA$ and a coherence length of $34 \AA$ has been observed. The schemes (Fig. 7c and d) shows the packing of 2DPP-OD-TEG and possible charge transport pathways such as the planar conjugated backbone direction and the $\pi-\pi$ stacking direction. It shows that the polymer adopts an edge-on orientation on the substrate where the $\pi-\pi$ stacking direction is parallel to the substrate. The shorter $\pi-\pi$ stacking distance (3.6 $\AA$ ) and large coherence length (34 ̊) of $\pi-\pi$ stacking interactions is attributed to the strong intermolecular interactions of the neighboring polymer chains and the presence of larger polymer crystallites with long range lattice ordering. Enhanced transport properties are expected for such films exhibiting long range order and films with a short $\pi-\pi$ stacking distance.
GIXD experiments were performed to understand the influence of solubilizing chains in DPP polymers on the resultant thin film morphology. Fig. 8a shows the GIXD pattern and corresponding line cuts of 2DPP-OD-HE spin coated onto Si substrates. The diffractogram shows that the diffractions align vertically along the (h00) Bragg peaks, which display a lamellar texture that adopts an edge-on orientation. The reflections associated with the $\pi-\pi$ stacking are most intense along the $Q_{x y}$ plane, which shows that the orientation of this stacking is mostly parallel to the substrate. The lamellae (h00) are associated with a $d$-spacing of $20 \AA$ (Fig. 8a), and the in-plane $\pi-\pi$ stacking (010) peak has a $d$-spacing of $3.6 \AA$. Branching is introduced in 2DPP-OD-EH with 2-ethyl hexyl chains, and Fig. $8 \mathrm{~b}$ shows that branching increases the misalignment of the polymer, as a significant arching of these peaks can be seen, indicating that the crystallites are no longer completely aligned perpendicular to the substrate, but are more randomly oriented. Fig. 8c shows a thin film GIXD pattern of 2DPP-OD-OD which exhibits a dual texture of face-on and edge-on alignment as the (h00) reflection shows both out-of-plane and in-plane orientation.

\section{Density Functional Theory (DFT) calculations}

Electronic structure and molecular geometry were investigated with density functional theory (DFT). Monomers through tetramers of the DPP were optimized at the B3LYP/6-31G* level of theory. 


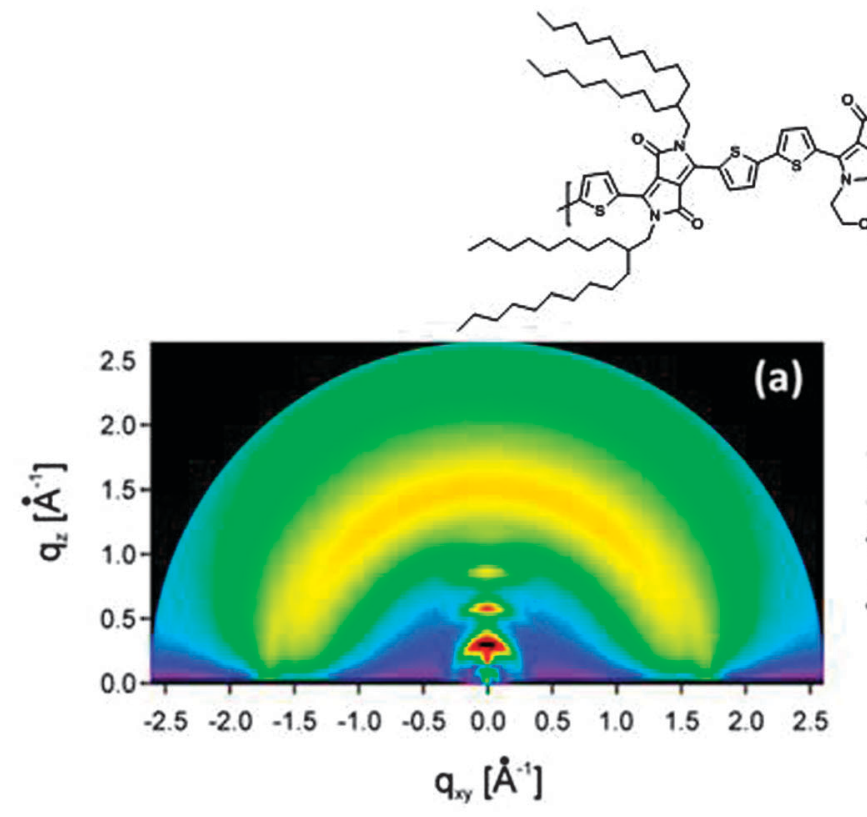

(c)
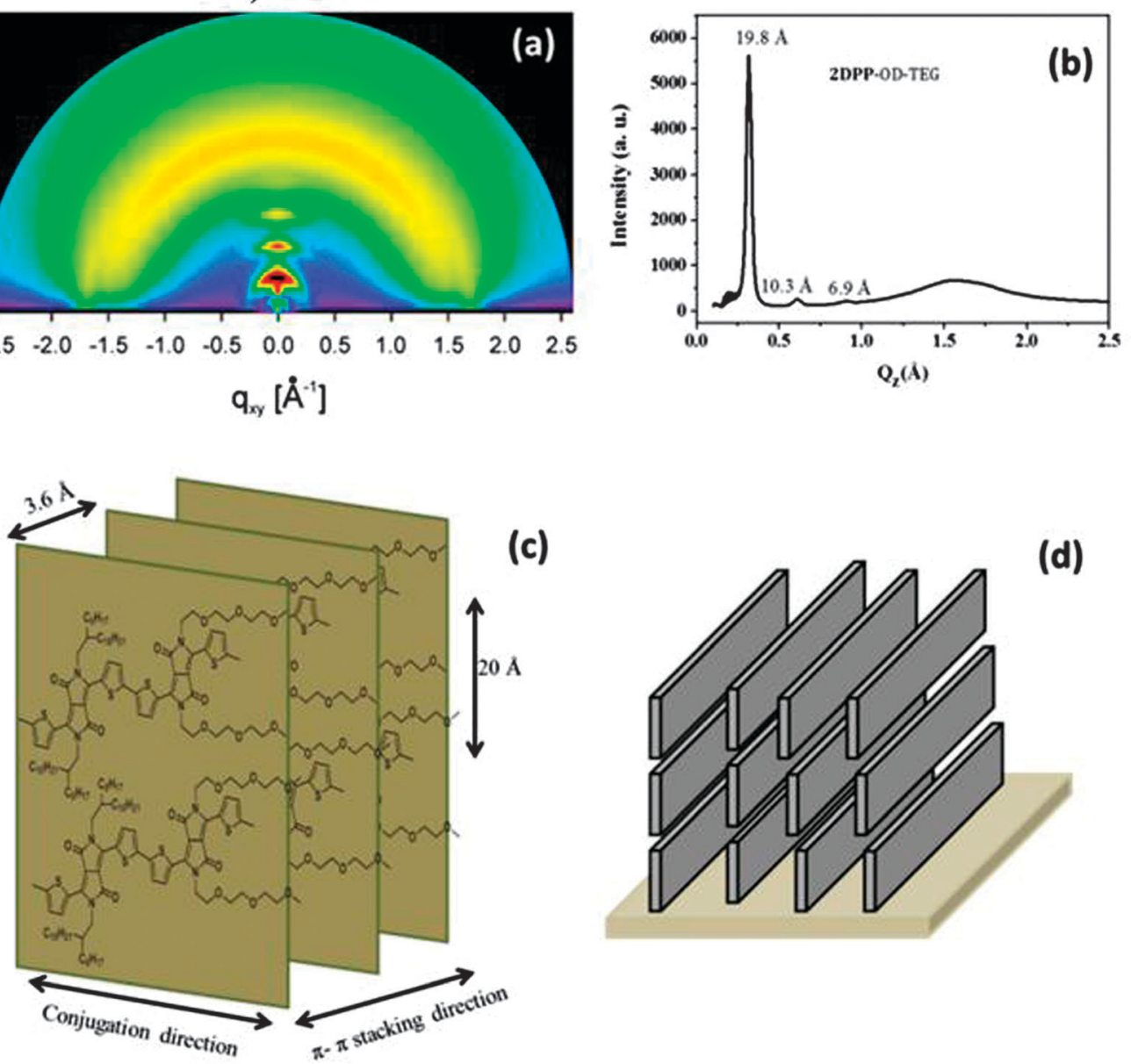

Fig. 7 (a) GIXD pattern, (b) intensity profile, (c) scheme for possible orientation of polymer chains and (d) edge-on orientation of as-spun 2DPP-OD-TEG thin films. Fig. 7a adapted from ref. 21 with permission from ACS.

Octadecyl side groups were replaced with methyl groups. For the $\mathrm{R}$ group (compared in Scheme 1), the TEG and methyl groups were tested. Because no differences between electronic structures of oligomers with TEG and methyl side-groups were found, all further calculations were done with methyl groups. The side-chains do not influence the electronic properties in the gas phase, confirming that the effects of different substituents occur through changes in morphology. UV-spectra were calculated with time-dependent DFT at TDB3LYP/6-31G*. All calculations were done with Gaussian 09. ${ }^{32}$

The oligomers are planar even in the gas phase, allowing for optimal conjugation along the chain. The four highest occupied and the four lowest unoccupied molecular orbitals of 4DPP are shown in Fig. S4 (ESI $\dagger$ ). In spite of the (thiophene) donor and (DPP) acceptor character, the electron densities of the HOMO and LUMO are delocalized over donor and acceptor units. Only a slight tendency towards localization is observed for the lower lying occupied orbitals. This is in contrast to benzothiadiazole (BDT) copolymers where the LUMO is mainly localized on the BDT units. ${ }^{33}$ As a result of the delocalized nature of the orbitals, the valence and conduction bands of the polymer, as obtained by extrapolation of the band edges (Fig. 9), are both wide. In stark contrast to copolymers containing BDT, the conduction band is wider than the valence band. ${ }^{34}$ Thus, theory predicts higher intramolecular electron than hole mobility, in agreement with the experimentally observed high ambipolar mobility in OFETs.

Charges in the individual thiophene and DPP rings were evaluated using NBO analysis. In the ground state, DPP units are negatively charged with -0.15 e, reflecting the donor-acceptor 


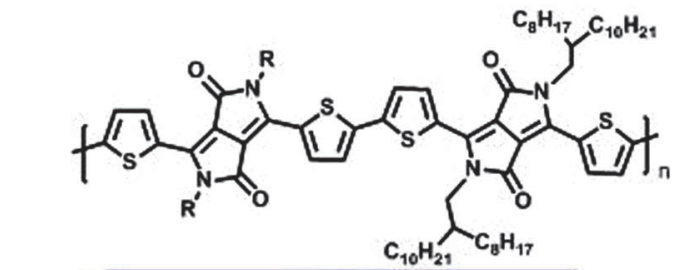

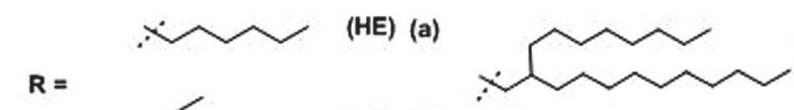
(OD) (c)
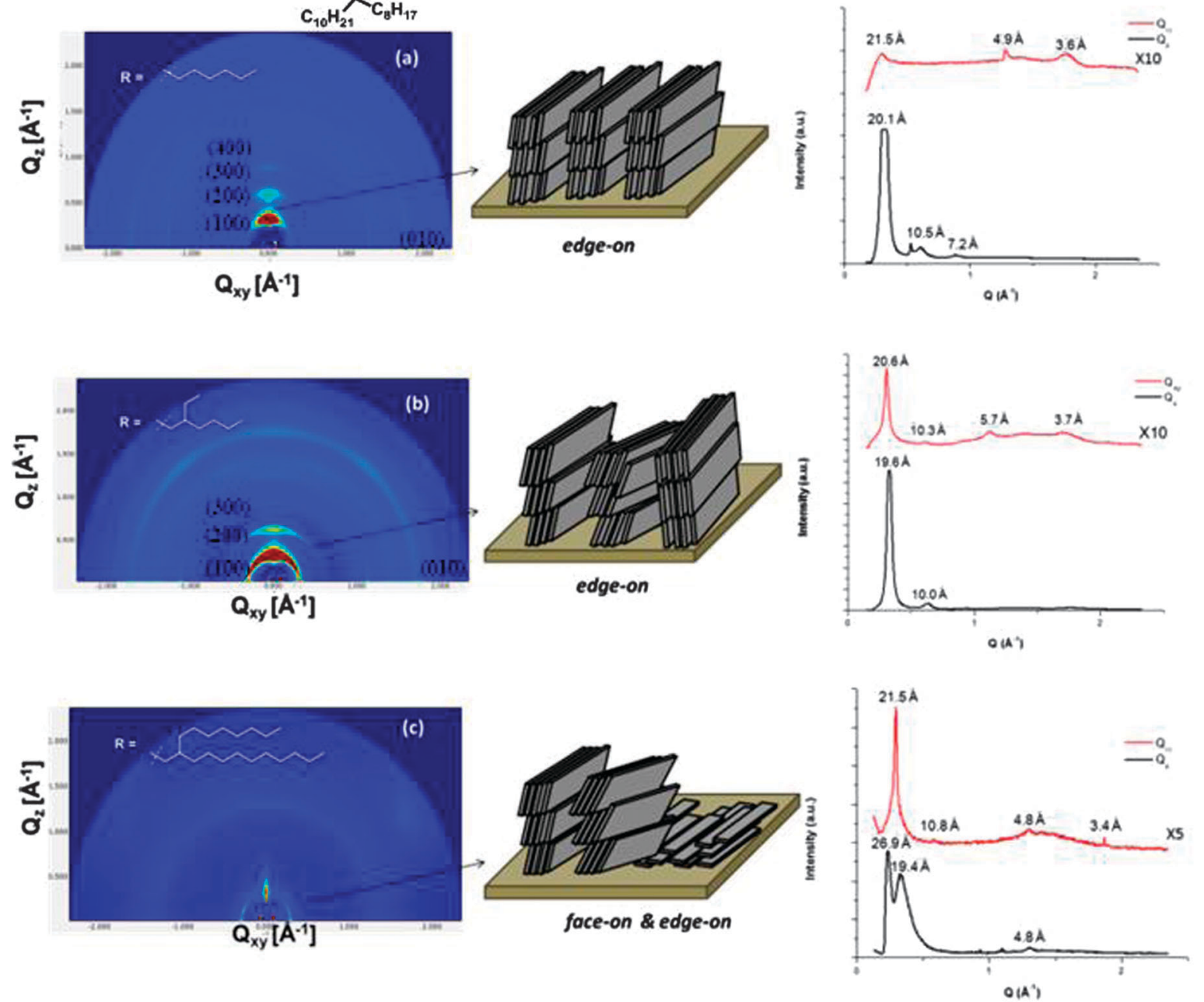

Fig. 8 GIXD pattern and intensity profiles of as-spun 2DPP-OD-HE (a), 2DPP-OD-EH (b) and 2DPP-OD-OD (c) thin films.

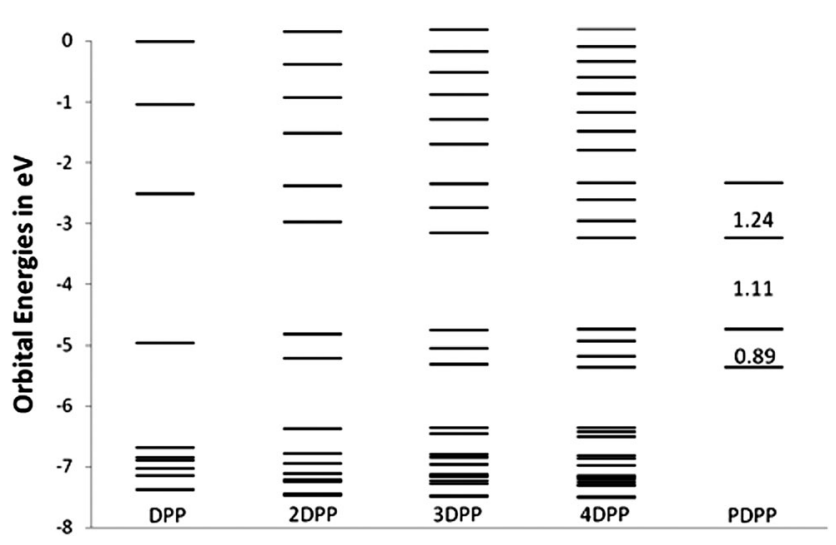

Fig. 9 Orbital energies of 1DPP-4DPP and bands of PDPP. character of the system. Upon excitation, however, the charge decreases to -0.14 e. Thus, there is practically no charge transfer upon excitation and the small charge transfer that does occur, shifts electron density from DPP to thiophene. As a result, the low energy band does not have charge transfer character; it is rather a $\pi-\pi^{*}$ transition similar in nature to those in observed in homopolymers. This is again in contrast to BDT copolymers where a charge transfer of 0.16 e from thiophene to BDT occurs upon excitation.

The electronic spectra of 1DPP-4DPP in Fig. 10 demonstrate that there is a strong red-shift accompanied by a large increase in oscillator strength upon chain length extension. This is evidence for strong conjugation along the polymer backbone. The weak absorptions at higher energy are red-shifted, but change very little in intensity upon chain length increase. Analysis of the electron configurations contributing to the bands reveals that the strong 


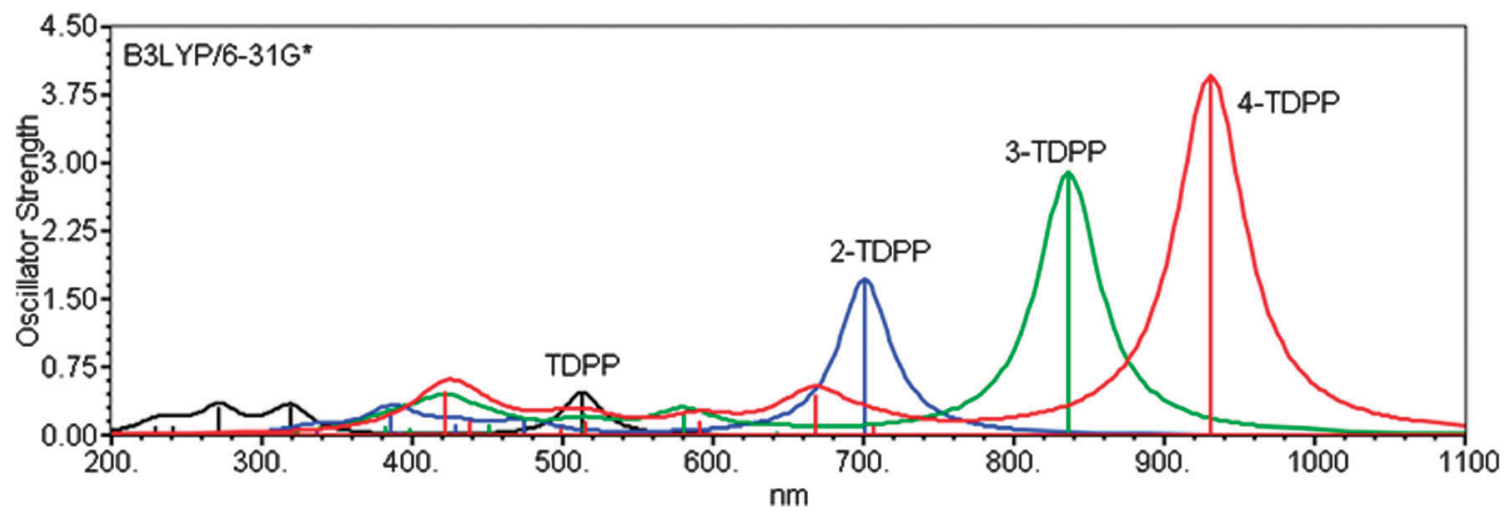

Fig. 10 UV-spectra of 1DPP-4DPP at B3LYP/6-31G*.

low energy absorption is a HOMO-LUMO $\pi-\pi^{*}$ transition, and the higher energy absorptions involve HOMO -1 through HOMO -3 and LUMO +1 through LUMO +3 , as in the case of homopolymers.

In summary, theoretical analysis reveals that DPP-DPP copolymers differ significantly from their BDT analogues, although the LUMO of DPP lies only $0.05 \mathrm{eV}$ higher in energy than that of BDT. All theoretical and experimental results are in agreement that DPP copolymers behave more like homopolymers with a single, strong, low-energy absorption. The exceptional electron mobility in this system is a result of the strong interaction between donor and acceptor in ground and excited states, that is reflected in the wide conduction band and the large red-shift and high oscillator strength of the low-energy absorption.

\section{Organic field-effect transistor measurements}

The copolymers were tested using (1) bottom-gate bottomcontact (BG-BC) and (2) top-gate bottom-contact (TG-BC) transistor configurations. BG-BC devices were made from $\mathrm{Si} / \mathrm{SiO}_{2}$ substrates with pre-patterned Au source/drain electrodes. The surface of $\mathrm{SiO}_{2}$ was treated with hexamethyldisilazane (HMDS) in order to passivate its surface and prevent formation of charge trapping states. The polymer semiconductor layer was spun on top of the substrates to complete the transistor fabrication, followed by thermal annealing at $140{ }^{\circ} \mathrm{C}$ in a nitrogen atmosphere. TG-BC transistors were fabricated on glass substrates containing pre-patterned Al source/drain electrodes. $^{35}$ The semiconducting polymer solution was then spun-cast onto the substrates at room temperature in a nitrogen atmosphere, followed by thermal annealing at $140{ }^{\circ} \mathrm{C}$. The insulating fluoropolymer CYTOP (Ashai Glass) was then sequentially spun-cast directly onto the semiconducting polymer at room temperature followed by annealing at $100{ }^{\circ} \mathrm{C}$ for 30 minutes. Device fabrication was completed with the evaporation of the Al gate electrodes by thermal evaporation under high vacuum $\left(10^{-6} \mathrm{mbar}\right)$.

The BG-BC transistor based on 2DPP-OD-TEG exhibited clear ambipolar characteristics (Fig. 11) with electron and hole mobilities in the order of $10^{-2} \mathrm{~cm}^{2} \mathrm{~V}^{-1} \mathrm{~s}^{-1}$. The extracted device parameters are summarized in Table 2. Charge carrier
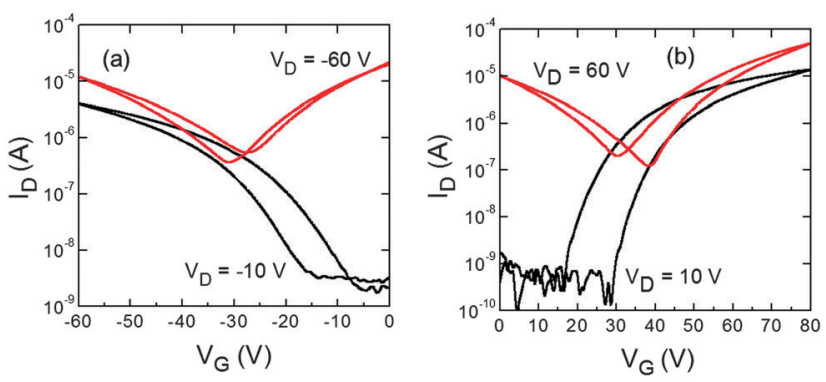

Fig. 11 Transfer characteristics obtained at negative $V_{G}$ (a) and positive $V_{G}$ (b) from a BG-BC transistor based on the 2DPP-OD-TEG polymer.

mobility in the saturated operating regime was extracted using eqn (1):

$$
I_{\mathrm{DS}}=\frac{W \mu_{\mathrm{FE}} C_{\mathrm{ox}}}{L 2}\left(V_{\mathrm{G}}-V_{\mathrm{t}}\right)^{2}
$$

where, $I_{\mathrm{DS}}$ is the source-drain current, $V_{\mathrm{G}}$ is the applied gate voltage, $V_{\mathrm{t}}$ is the threshold voltage of the device, $W$ is the channel width, $L$ is the channel length, $C_{\text {ox }}$ is the capacitance of the oxide layer and $\mu_{\mathrm{FE}}$ is the field-effect mobility. The threshold voltage was extracted from the transfer characteristics measured with $V_{\mathrm{D}}=|60 \mathrm{~V}|$ and from the intersection of the extrapolated linear part of the $I_{\mathrm{DS}}{ }^{1 / 2}$ with the $V_{\mathrm{G}}$ axis. The on/off ratio for the transistors was also calculated yielding values in the order of $10^{4}$.

Fig. 12 displays the output and transfer characteristics of BG-BC transistors based on 2DPP-OD-HE and 2DPP-OD-EH copolymers. Unfortunately, no measurable channel current was obtained from transistors based on 2DPP-OD-OD. On the other hand, transistors based on the 2DPP-OD-HE and 2DPPOD-EH copolymers show clear transistor action exhibiting hole mobility in the order of $10^{-4} \mathrm{~cm}^{2} \mathrm{~V}^{-1} \mathrm{~s}^{-1}$. Although similar charge carrier mobilities are obtained from the two polymers incorporating $n$-hexyl and 2-ethyl hexyl side chains, a large difference in the threshold of the two types of devices can be observed $-V_{\text {th }} \sim-15 \mathrm{~V}$ and $V_{\text {th }} \sim-27 \mathrm{~V}$ for 2DPP-OD-HE and 2DPP-OD-EH based transistors, respectively. Since $V_{\text {th }}$ is a measure of charge trapping sites present at the dielectric/polymer interface (i.e. the semiconducting channel), the difference in the 
Table 2 Summary of OFET device characteristics

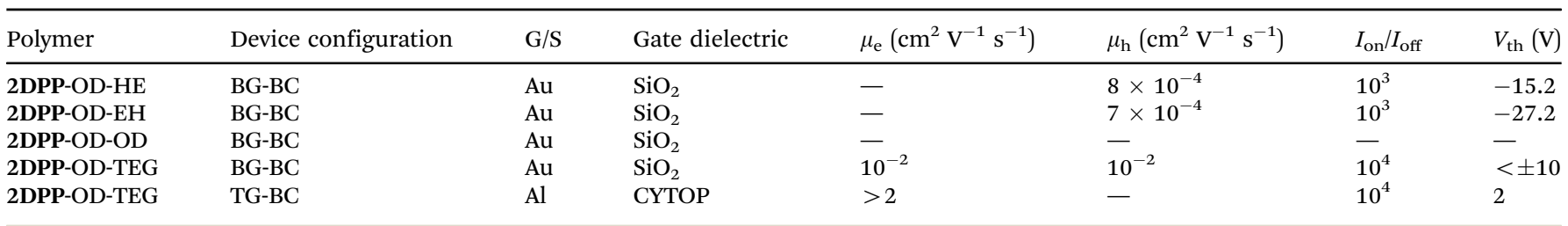
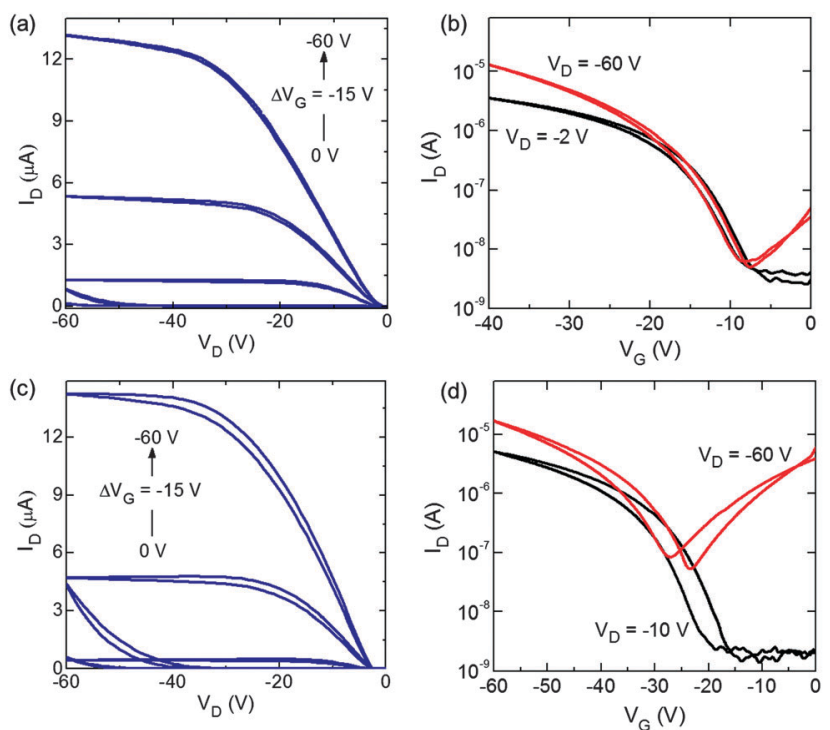

Fig. 12 Output and transfer characteristics measured from BG-BC transistors based on the copolymer 2DPP-OD-HE [(a) and (b), respectively] and the 2DPP-OD-EH copolymer [(c) and (d), respectively].

$V_{\text {th }}$ most likely indicates the presence of a larger concentration of traps in the case of 2DPP-OD-EH devices, possibly due to branching which introduces increases in structural, and hence energetic, disorder in the solid film as compared to devices based on 2DPP-OD-HE. The polymer with triethylene glycol chains (2DPP-OD-TEG), on the other hand, shows low $V_{\text {th }}$ and higher charge carrier mobility (Table 2).

Fig. 13 displays a representative set of the transfer characteristics obtained from a TG-BC transistor based on the 2DPPOD-TEG copolymer. The devices show high channel currents as a direct result of the high electron mobility, which for some devices exceeds $2( \pm 0.5) \mathrm{cm}^{2} \mathrm{~V}^{-1} \mathrm{~s}^{-1}$. The channel current on/off ratio is also high and typically in the order of $10^{4}$ or higher. The reason for this dramatically enhanced electron mobility is believed to be the extended $\pi$-conjugation in combination with the long range structural order (lamellar packing); evidence for the latter is provided from the GIXD measurements. This unique combination of excellent intramolecular and intermolecular transport in the solid film leads to a dramatically enhanced electron mobility, as compared to the other copolymers. All four polymers were tested in TG-BC architecture. Unfortunately, only devices based on the 2DPPOD-TEG polymer, showed transistor function, with the rest of the polymers resulting in non-functioning transistors, i.e. typically devices exhibiting high gate leakage currents,

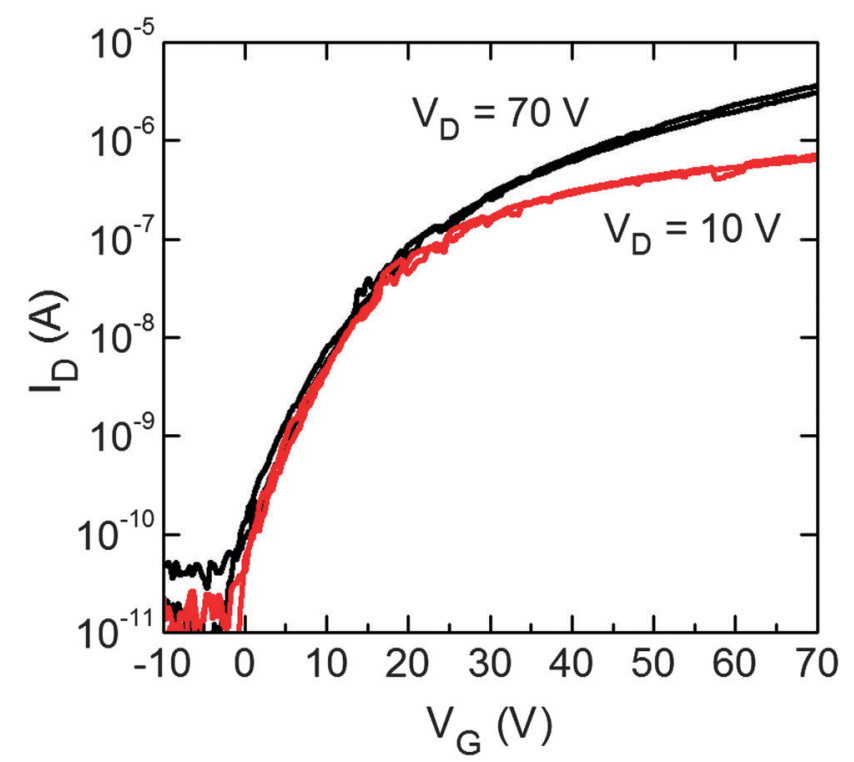

Fig. 13 Transfer characteristics measured from a TG-BC transistor based on the 2DPP-OD-TEG copolymer. Adapted from ref. 21with permission from ACS.

most likely due to the non-optimized morphology of the polymer/ dielectric interface.

In the case of the TG-BC transistors based on the 2DPP-OD-TEG copolymer, the low work function $\mathrm{Al}(4.3 \mathrm{eV})$ source-drain electrodes were replaced with high work function gold $(\mathrm{Au} \sim 5 \mathrm{eV})$ electrodes. As-evaporated $\mathrm{Au}$ electrodes were treated with the contact work function modifier pentafluorobenzene (PFBT). A very interesting observation is that as opposed to the BG-BC transistor characteristics shown in Fig. 11, the TG-BC devices exhibit no hole transporting characteristics (Fig. 14) and exhibit clear unipolar electron transport character even when the source/drain electrodes are replaced with the high work function, and hence the hole injecting Au electrodes.
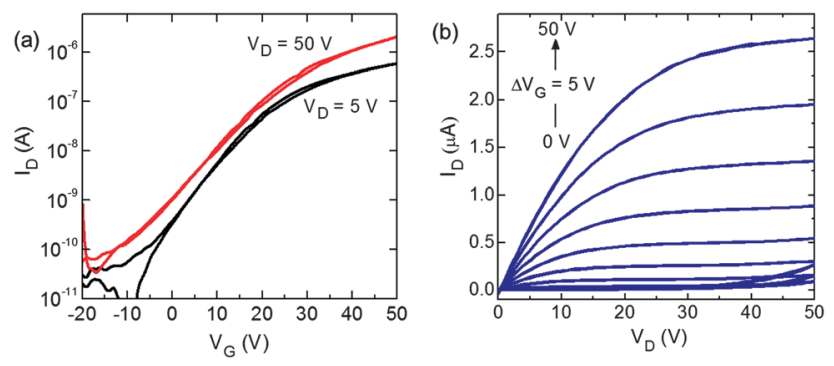

Fig. 14 Transfer (a) and output (b) characteristics measured for TG-BC transistors based on 2DPP-OD-TEG. 


\section{Summary and conclusions}

In summary, we have developed a novel series of alternating copolymers by coupling electron-deficient DPP-based monomers. The coupling of diketopyrrolopyrrole with diketopyrrolopyrrole in an alternate fashion affords remarkable improvement in optical properties and ambipolar charge carrier mobility. In this contribution, we also emphasized the critical role of substituted alkyl chains on the charge transport properties, thin film morphology, and electronic properties of these newly developed molecular semiconductors. The results obtained suggest that a better electronic structure or the position of LUMO energy is not the only decisive factor for improving charge carrier mobility; the superior morphology of the polymer thin films and the conformation induced by substituted side chains also contributes significantly to the solid state packing and the charge transport. The electronic structure, thin film morphology, and molecular packing obtained from GIXD measurements present a detailed understanding of charge transport properties of this emerging class of molecular semiconductors. The novel synthetic design approach and demonstration of organic field-effect transistors (2DPP-OD-TEG) with electron mobilities $>2 \mathrm{~cm}^{2} \mathrm{~V}^{-1} \mathrm{~s}^{-1}$ further validates the potential of these DPP-based conjugated polymers. We also make a note that the molecular weight has a strong influence on the charge carrier mobilities of these polymers.

\section{Acknowledgements}

The authors acknowledge financial support from the Department of Science and Technology, India through the Indo-UK Apex Program and Ministry of Communication and Information Technology under a grant for the Centre of Excellence in Nanoelectronics, Phase II. E. K. B. and A. L. B. acknowledge the National Science Foundation for their support (DMR-1112455). GIXD was carried out at Stanford Synchrotron Light Source (SSRL).

\section{References}

1 D. Choi, B.-S. Jeong, B. Ahn, D. S. Chung, K. Lim, S. H. Kim, S. U. Park, M. Ree, J. Ko and C. E. Park, ACS Appl. Mater. Interfaces, 2011, 4, 702.

2 A. R. Postema, K. Liou, F. Wudl and P. Smith, Macromolecules, 1990, 23, 1842.

3 J. Luo, S. Liu, M. Haller, L. Liu, H. Ma and A. K. Y. Jen, Adv. Mater., 2002, 14, 1763.

4 S. Hotta, S. D. D. V. Rughooputh, A. J. Heeger and F. Wudl, Macromolecules, 1987, 20, 212.

5 K.-Y. Jen, G. G. Miller and R. L. Elsenbaumer, J. Chem. Soc., Chem. Commun., 1986, 1346.

6 P.-T. Wu, H. Xin, F. S. Kim, G. Ren and S. A. Jenekhe, Macromolecules, 2009, 42, 8817.

7 X. Guo, F. S. Kim, S. A. Jenekhe and M. D. Watson, J. Am. Chem. Soc., 2009, 131, 7206.

8 N. S. Sariciftci, L. Smilowitz, A. J. Heeger and F. Wudl, Science, 1992, 258, 1474.

9 J. Chen and Y. Cao, Acc. Chem. Res., 2009, 42, 1709.
10 J. Li, Y. Zhao, H. S. Tan, Y. Guo, C.-A. Di, G. Yu, Y. Liu, M. Lin, S. H. Lim, Y. Zhou, H. Su and B. S. Ong, Sci. Rep., 2012, 2, 754 .

11 F. Eder, H. Klauk, M. Halik, U. Zschieschang, G. Schmid and C. Dehm, Appl. Phys. Lett., 2004, 84, 2673.

12 L.-L. Chua, J. Zaumseil, J.-F. Chang, E. C. W. Ou, P. K. H. Ho, H. Sirringhaus and R. H. Friend, Nature, 2005, 434, 194.

13 A. R. Murphy and J. M. J. Fréchet, Chem. Rev., 2007, 107, 1066.

14 H. Usta, C. Risko, Z. Wang, H. Huang, M. K. Deliomeroglu, A. Zhukhovitskiy, A. Facchetti and T. J. Marks, J. Am. Chem. Soc., 2009, 131, 5586.

15 D. M. de Leeuw, M. M. J. Simenon, A. R. Brown and R. E. F. Einerhand, Synth. Met., 1997, 87, 53.

16 C. R. Newman, C. D. Frisbie, D. A. da Silva Filho, J.-L. Brédas, P. C. Ewbank and K. R. Mann, Chem. Mater., 2004, 16, 4436.

17 C. Kim, A. Facchetti and T. J. Marks, Science, 2007, 318, 76. 18 T. Lei, J.-H. Dou, X.-Y. Cao, J.-Y. Wang and J. Pei, J. Am. Chem. Soc., 2013, 135, 12168.

19 Y. Zhao, Y. Guo and Y. Liu, Adv. Mater., 2013, 25, 5372.

20 C. B. Nielsen, M. Turbiez and I. McCulloch, Adv. Mater., 2013, 25, 1859.

21 C. Kanimozhi, N. Yaacobi-Gross, K. W. Chou, A. Amassian, T. D. Anthopoulos and S. Patil, J. Am. Chem. Soc., 2012, 134, 16532.

22 M. A. Naik, N. Venkatramaiah, C. Kanimozhi and S. Patil, J. Phys. Chem. C, 2012, 116, 26128.

23 C. D. Dimitrakopoulos and P. R. L. Malenfant, Adv. Mater., 2002, 14, 99.

24 A. C. Mayer, M. F. Toney, S. R. Scully, J. Rivnay, C. J. Brabec, M. Scharber, M. Koppe, M. Heeney, I. McCulloch and M. D. McGehee, Adv. Funct. Mater., 2009, 19, 1173.

25 H. Burckstummer, A. Weissenstein, D. Bialas and F. J. Wurthner, J. Org. Chem., 2011, 76, 2426.

26 L. Huo, J. Hou, H.-Y. Chen, S. Zhang, Y. Jiang, T. L. Chen and Y. Yang, Macromolecules, 2009, 42, 6564.

27 W. Li, W. S. C. Roelofs, M. M. Wienk and R. A. J. Janssen, J. Am. Chem. Soc., 2012, 134, 13787.

28 H. Bronstein, Z. Chen, R. S. Ashraf, W. Zhang, J. Du, J. R. Durrant, P. Shakya Tuladhar, K. Song, S. E. Watkins, Y. Geerts, M. M. Wienk, R. A. J. Janssen, T. Anthopoulos, H. Sirringhaus, M. Heeney and I. McCulloch, J. Am. Chem. Soc., 2011, 133, 3272.

29 J. C. Bijleveld, V. S. Gevaerts, D. Di Nuzzo, M. Turbiez, S. G. J. Mathijssen, D. M. de Leeuw, M. M. Wienk and R. A. J. Janssen, Adv. Mater., 2010, 22, E242.

30 Y. H. Kim, S. K. Park, D. G. Moon, W. K. Kim and J. I. Han, Jpn. J. Appl. Phys., 2004, 43, 3605.

31 S. Ando, J.-i. Nishida, H. Tada, Y. Inoue, S. Tokito and Y. Yamashita, J. Am. Chem. Soc., 2005, 127, 5336.

32 M. J. Frisch, T. G. W. Trucks, H. B. Schlegel, G. E. Scuseria, M. A. Robb, J. R. Cheeseman, G. Scalmani, V. Barone, B. Mennucci, G. A. Petersson, H. Nakatsuji, M. Caricato, H. P. Hratchian, A. F. Izmaylov, J. Bloino, G. Zheng, J. L. Sonneberg, M. Hada, M. Ehara, K. Toyota, R. Fukuda, 
J. Hasegawa, M. Ishida, T. Nakajima, Y. Honda, O. Kitao, H. Nakai, T. Vreven, J. A. Montgomery Jr., J. E. Peralta, F. Ogliaro, M. Bearpark, J. J. Heyd, E. Brothers, K. N. Kudin, V. N. Staroverov, R. Kobayashi, J. Normand, K. Raghavachari, A. Rendell, J. C. Burant, S. S. Iyengar, J. Tomasi, M. Cossi, N. Rega, J. M. Millam, M. Klene, J. E. Knox, J. B. Cross, V. Bakken, C. Adamo, J. Jaramillo, R. Gomperts, R. E. Stratmann, O. Yazyev, A. J. Austin, R. Cammi, C. Pomelli, J. W. Ochterski, R. L. Martin, K. Morokuma, V. G. Zakrzewski, G. A. Voth, P. Salvador, J. J. Dannenberg,
S. Dapprich, A. D. Daniels, O. Farkas, J. B. Foresman, J. V. Ortiz, J. Cioslowski and D. J. Fox, Gaussian 09, Revision A.1, Gaussian, Inc., Wallinford, CT, 2009.

33 G. L. Gibson, T. M. McCormick and D. S. Seferos, J. Am. Chem. Soc., 2011, 134, 539.

34 U. Salzner, O. Karaltı and S. Durdaği, J. Mol. Model., 2006, $12,687$.

35 J. Smith, W. Zhang, R. Sougrat, K. Zhao, R. Li, D. Cha, A. Amassian, M. Heeney, I. McCulloch and T. D. Anthopoulos, Adv. Mater., 2012, 24, 2441. 